\title{
Toward Cloud COMPUTING: SECURITY AND PeRformance
}

\author{
Nabeel Zanoon \\ Department of applied science, Al- Balqa' Applied University, Jordan, Amman
}

\begin{abstract}
Security and performance are basic requirements for any system. They are considered the criteria for the measurement of any progress in a security system. Security is an indicator that affects the level of performance through the threats that influence the performance of parts of the cloud during the rendering of services. Both security and performance demonstrate the efficiency of cloud computing which indicates that the performance and security are measurements for the extent of the development of the cloud. In this paper, the relationship between performance and security will be examined to know the extent of their impact on the progress of cloud computing.
\end{abstract}

\section{KEYWORDS}

Cloud Computing, Security and Performance, SLA, Distributed computing

\section{INTRODUCTION}

Cloud computing is a new technology in the world of information as the development of distributed computing derives from parallel computing, network computing and distributed databases [1]. If we want to analyze the term computerized networks, we say that they are a group of computers distributed to geographical areas and are connected together by a mediator. However, computerized networks are a term which indicates that the devices are distributed individually and are connected through the exchange of messages [2]. Distributed computing is a technology which links the devices together individually across geographical areas that constitute a single environment and share the resource. All the devices are connected together to implement several tasks [3.] The cloud develops with the progress of the public Internet, because the cloud capabilities are limited in order to provide security on the basis of performance. Most of the companies that seek to take advantage of cloud services follow the swiftness policy on the one hand, and security and performance on the other hand, for it renounces its security through contact on public cloud services in which case default resources are obtained, and this exposes the enterprise content and data to most of the Internet vulnerabilities [4]. Security and privacy constitute a tremendous agility in the development of the cloud. Security and privacy are to be provided by cloud service providers as well as the reliability, availability, and performance [5]. For the companies that specialize in information technology, which provide the cloud services, the availability of performance and security are the challenges when adopting cloud computing by customers to make use of the services. The violation of security constitutes a risk of data loss, and also the level of performance could weaken because of limited bandwidth, disk space, memory, and the CPU cycle and network connection latency. Therefore, the level of performance in the cloud services is a factor in users' acceptance to the cloud [6].

The progress in the services that are provided through the cloud computing networks must be characterized by the high level of performance and security. The raising of the level of 
performance may be at the expense of the level of security where there is a relationship between them and this relationship has an influence on the progress and development of the cloud computing.

\section{Cloud Computing}

The cloud computing offers resource services in order to provide space for storage. The Email is an example that shows the concept of cloud computing where you use resources to store your messages, and these resources are not on your PC but on a resource somewhere else unknown to the user [7]. The cloud spreads on several levels, including the private, local, public and mixed, while the service models include (PAAS), (IAAS) and (SAAS) [8].

\subsection{Characteristics of Cloud Computing}

The mechanism of action of cloud computing is on-demand self-service. One can access the cloud through multiple devices such as cellular's, computers and workstations, where resource pooling is conducted by using a multi-tenant model, though the users cannot know where the available resources are. The cloud provides resources in a flexible and fast manner in line with consumer demand and while providing resources the cloud tracks the service measurement. The controlled cloud computing resources can be measured, and transparency can be provided for both the service provider and the consumer of the service used. Cloud computing services use measurement capabilities that enable control and optimal use of resources [9].

\section{The Performance of Cloud Computing}

Performance is an outlet for any system. A definition of performance has been proposed based on the ISO 25010 scale where the performance of cloud computing system is determined by analyzing the properties involved in the performance of an effective and reliable service to meet the needs mentioned under and within the maximum transaction system boundary conditions. Measures and standards are applied to the three elements of users, suppliers and supervisors. Figure (1) shows that each output system is based on several possibilities, that is, the system may provide a service correctly or incorrectly, or the service may be altogether rejected and this affects the performance of the system [10].

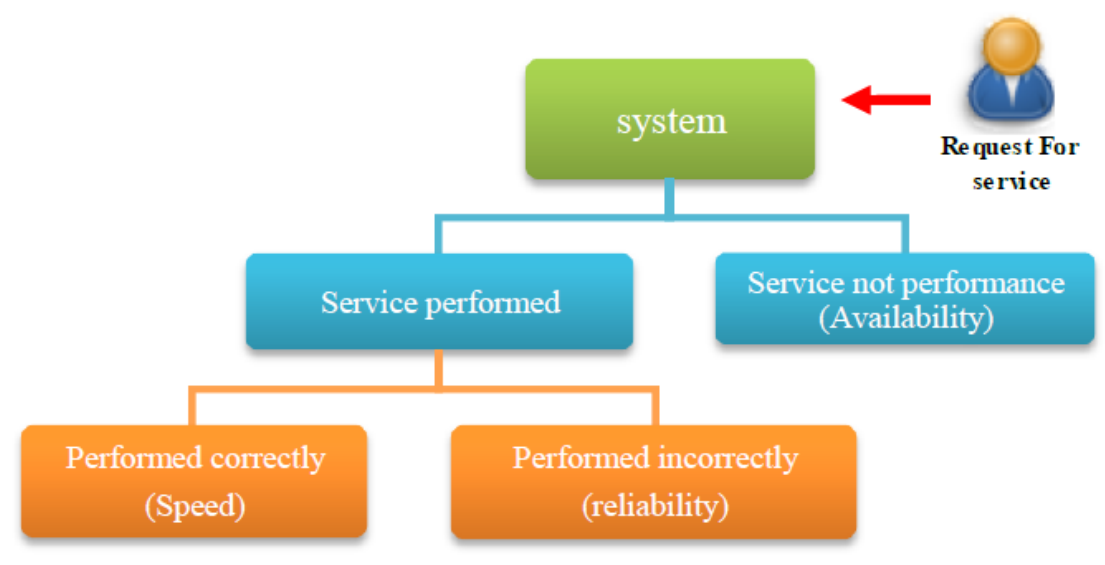

Figure 1. Computerized computing system outputs

Cloud computing is one of the distributed systems which deals with dispersed resources and is based on processing big size data and this need called for the performance control and analysis 
techniques to develop them, that is to say cloud control is important for both users and service providers. It provides information and indicators of performance, where that performance level affects the adoption of cloud services by users [11]. Performance is among the advantages that should be available in the cloud services because performance has an impact on users and service providers. To evaluate performance, we must take into account several criteria to evaluate the factors that affect the performance of cloud computing, including the average response per unit time and the average waiting time per unit time and others [12] in the properties of performance measures of the cloud.

- (SaaS) Evaluation is made by users directly depending on the performance measures, the speed of response, reliability of technical services and availability.

- (PaaS) Evaluation is made by users directly or indirectly depending on the performance measures in response to technicality, productivity, reliability, technical service and middleware capability.

- (IaaS) The performance measures are determined depending on the infrastructure performance, capacity, reliability, availability, and scalability [13].

\subsection{Performance Metrics}

There are several options for the measures of performance of the cloud computing by service providers: delay in service and accuracy. The performance and evaluation are measured depending on the responsiveness time, productivity, and timing in implementing tasks, that is, processing tasks in the appropriate period. There are other measures as in Table 1.

Table 1. The future of service performance in the cloud [14]

\begin{tabular}{|c|l|l|}
\hline Features & \multicolumn{1}{|c|}{ Description } & \multicolumn{1}{|c|}{ Metrics } \\
\hline Communication & $\begin{array}{l}\text { Exchange data between internal } \\
\text { service or external consumer in } \\
\text { cloud }\end{array}$ & $\begin{array}{l}\bullet \text { Packet loss Frequency } \\
\text { Connection on Error Rate } \\
\bullet \text { MPI Transfer bit/Byte Speed }\end{array}$ \\
\hline Computation & $\begin{array}{l}\text { The Computing Data or Task } \\
\text { processing in the cloud systems }\end{array}$ & $\begin{array}{l}\bullet \text { CPU load (\%) } \\
\bullet \text { Benchmark OP(FLOP) Rate } \\
\bullet \text { Instance Efficiency (\% CPU Peak) }\end{array}$ \\
\hline Memory & $\begin{array}{l}\text { Designed for rapid use of } \\
\text { temporarily store information that } \\
\text { may be obtained from slow- } \\
\text { access hard disk drive }\end{array}$ & $\begin{array}{l}\bullet \text { Mean Hit Time(s) } \\
\text { Memory bit /Byte speed } \\
\text { (MB/s,GB/s) }\end{array}$ \\
\hline Time & $\begin{array}{l}\text { Project completion within time } \\
\text { while maintaining quality is very } \\
\text { important for the success of } \\
\text { project }\end{array}$ & $\begin{array}{l}\bullet \text { Computation time } \\
\bullet \text { Communication time }\end{array}$ \\
\hline
\end{tabular}

The (SLA) agreement restricts customers and cloud service providers. The level of service or quality of service (QOS) must be taken into account. The service performance is characterized by the response time, productivity, availability, and security [15]. The Quality of Service (QoS) in the cloud indicates the level of performance and reliability, despite the fact that the characteristics of quality of service received attention before the evolution of cloud computing, performance, homogeneity and other standards [16]. Speed is one of the (QoS) features that have been designated by (EG 202 009-1). It is the performance standard which represents the time interval 
necessary to perform a function or average at which the function and security are achieved, that is, any service capability to ensure the confidentiality of a piece of information you worked with, exchanged or stored, the privacy of communications, authenticity and safety of exchanged or stored information, as well as the protection of the user and his communication means against any kind of threat, in addition to other qualities of accuracy, reliability, flexibility and ease of use [17]. There are several risks and threats facing users when they make use of the cloud services. They are increasing in the case when the service provider or resources exist outside the regional scope, that is, they are under different laws. In relation to the adoption of the cloud in higher education, there are some challenges such as security, performance, efficiency and control [18].

Figure (2) shows that the (IDC) analysis considers performance as the second largest case after security in the cloud environment. The performance metrics is based on applications capabilities, where the performance weakens due to a lack of resources, disk space, limited bandwidth, low CPU speed, memory, and network connections [18].

\section{Q: Rate the challenges/issues of the 'cloud'/on-demand model}

(Scale: 1 = Not at all concerned 5 = Very concerned)

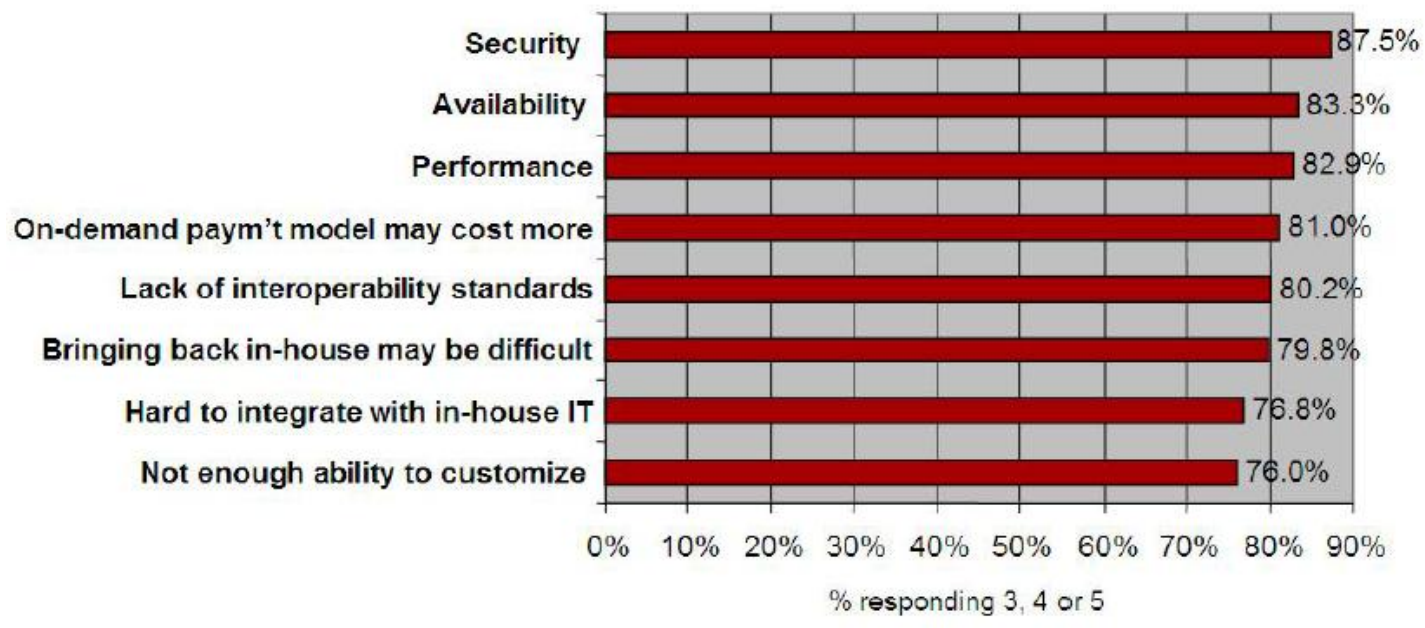

Figure 2. Rate of issues and challenges attributed to the cloud in the application form

\section{Security in Cloud Computing}

Security in the cloud computing is a highly sensitive and important factor, because the cloud deals with sensitive and confidential data in the cloud data centers. Therefore, protection and integrity of the data is the duty of providers of cloud services. Privacy and the security of cloud computing depend on the cloud service provider to put strong security controls and the policy of sound privacy to protect the data of its customers. This is because customers need confidence and transparency in terms of performance and security [19]. The cloud providers offer services to several organizations located in the same network, which grants access to the data without permission by another organization working in the same field of cloud data centers. In addition to that, physical security Judgments from the cloud service provider are regarded a source of concern [20].

The data protection in the cloud is an important security issue and should be taken into account in this issue in terms of the way they are accessed, stored, audit requirements and the cost of data 
breaches [21]. Privacy is one of the issues of security in the cloud, and so specialists in the development of the security services and practices of privacy in cloud services are a must. An effective evaluation strategy which covers data protection, compliance, privacy, and identity management, secure operations, and other relevant legal issues must be put forward [22]. There are concerns about privacy and security associated with the breach of privacy, access and use of information stored in the cloud for malicious purposes, and this is up to organizations that provide the services [23].

The cloud computing determines the characteristics and performance metrics between providers and customers through Service Level Agreements (SLAs), which are concerned with the legal basis for the services and the level of quality of the service [24]. Non-compliance with Service Level Agreement (SLA) by this cloud service providers leads to the slow rate of adoption and to a decline in the level of performance, in addition to concerns about the security and confidentiality of the data stored in the cloud, as well as privacy [25]. The Service Level Agreements are describe different measure scales on the basis of Quality of Service (QoS) [26]. The cloud proceeds, but this progress is confronted by obstacles in the field of Quality of Service (QoS) which is an indicator of the levels of performance, reliability, and availability of security [27].

Performance is wide; it includes reliability, energy efficiency and expansion. The cloud computing environments are also wide and combine multiple providers and users resources. Here, there is the security factor affecting the performance and through the security impact on the network infrastructure as, for example, is the case with the DDoS attacks which have a broad impact on network performance. If this risk or any risks that threaten the cloud environment itself, they will be a major concern for users and providers [28]. These attacks are harmful to the computing. Inoculating SQL attack allows the attacker access to the database. Also, in flood attacks the attacker sends a request for resources to the cloud so quickly that he takes advantage of the ability of any inaccessible resources by ordinary users and here many attacks that threaten the security of the cloud happen [29]. Security is an important priority to the cloud customers. The decision to make use of cloud services will be based on the level of confidentiality, integrity, flexibility, and security services available, and this is an indication to the competition among the service providers that leads to the development of cloud computing [30].

There are some problems facing the cloud computing users, in terms of access to the data through the Internet; any weakness in the level of security in the cloud is threatening the confidentiality of users data stored. Moreover, the quality of the connection affects the level of performance in the delivery of services, the high cost of private computerized computing compared with public and mixed sectors, at the expense of quality, performance and security [31].

\section{The Relationship Between Performance and Security}

The developing scale in any computerized system is based on the level of performance of the system, but there is a relationship between security and performance. Performance is an indicator to speed of implementation and exchange of data while security indicates the protection from threats. Both depend on the components of the system software and hardware on the one hand, and on the user and service provider on the other hand. Security is considered one of efficiency standards where efficiency is the main indicator of the level of performance, while the performance is an auxiliary element in the protection of the system. As a corollary, the relationship between performance and security is complementary to each and is a key factor to the development of the system.

Cloud computing provides services to users through service providers; the user submits a service request, where the service provider responds by providing the service. However, this process is based on an agreement between the two parties, in the light of which contact and making use are 
made. This agreement is called Service Level Agreement (SLA). Moreover, there are other inevitable issues between the user and the service provider, such as security and performance of the service which are factors that affect the acceptance of the user to make use of cloud services.

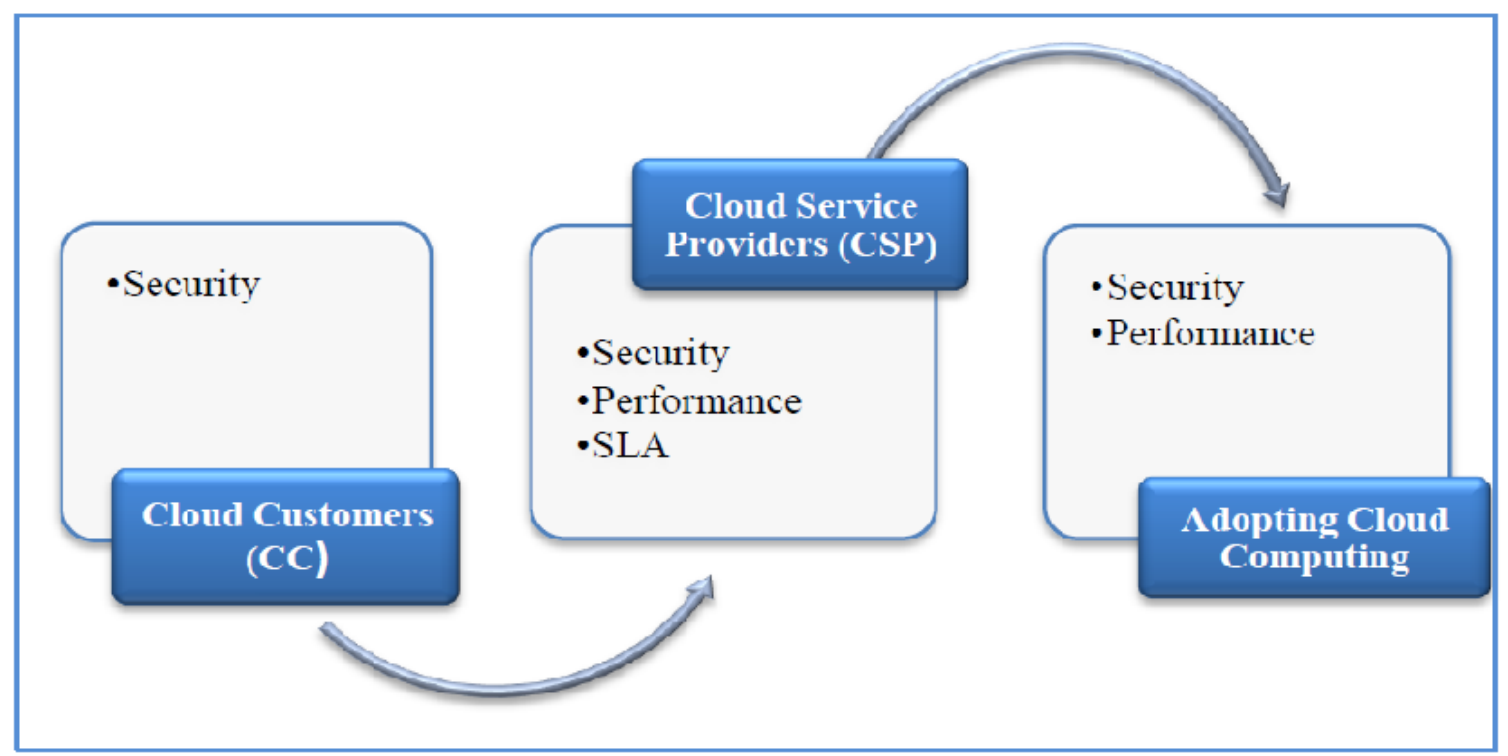

Figure 3. The relationship between performance and security in the cloud

Figure 3 shows the relationship between security and performance in the cloud. Security and performance are prerequisites for the user, and therefore the service provider is obliged to make it available to the user, while the performance on the part of the user is not obligatory because the user does not know where the data is stored and has no control over the data. Nevertheless, security is imperative to the user where the user represents a security breach in the cloud in case he did not the follow security policy, that is, the user may cause the low level of security and this leads to a low level performance of services. Also, if the low level of security affects performance, and according to the performance scale, the performance will influence the security on the part of the service provider.

In light of this we conclude that security and performance represent challenges in the cloud because each is a challenge to the other. Better security means better and better performance is better security which means that the relationship is a positive one. Performance and security for the service are a requirement at the user and provider level, while the data are on the provider and agreement level. This is because the user does not known the storage place and does not have control over the data as in Figure (4) 


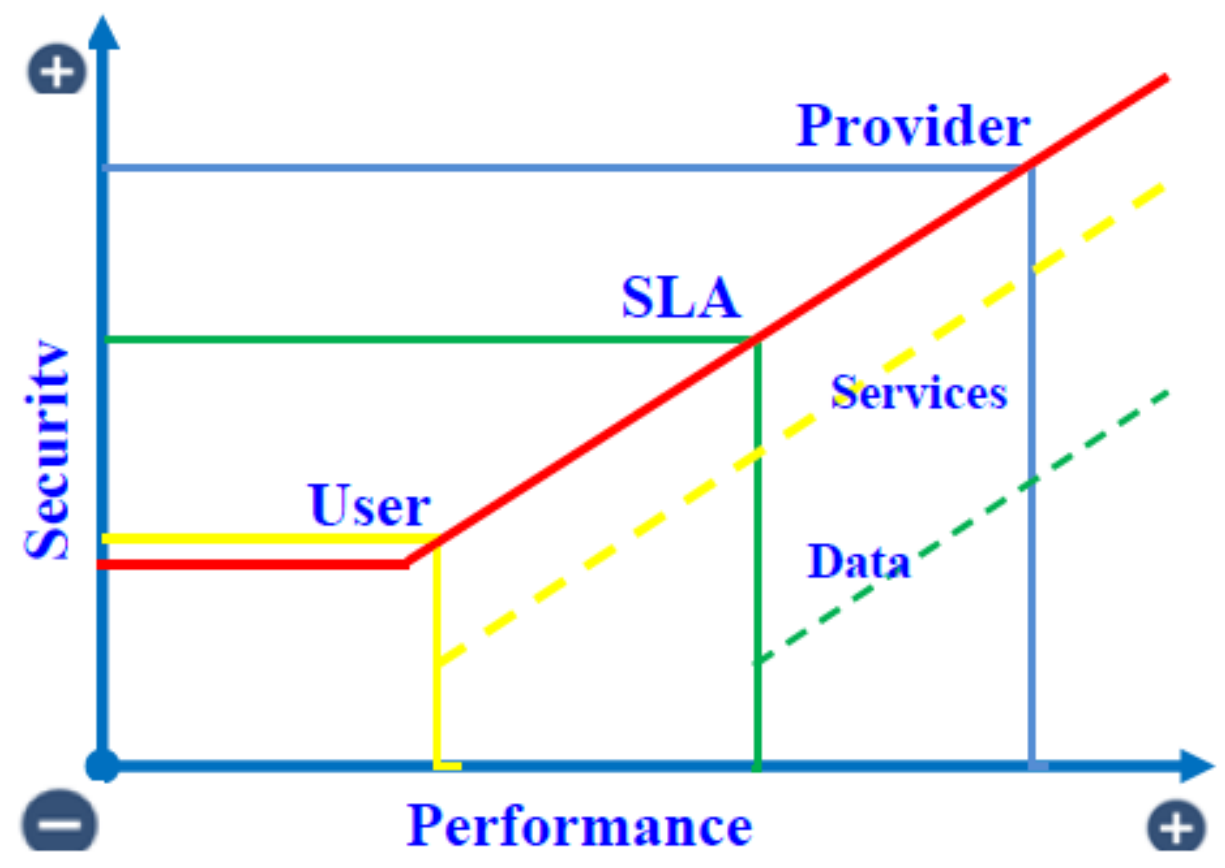

Figure 4. The level of impact between performance and security in cloud computing

\section{Conclusion}

The relationship between performance and security is a positive one. Security affects performance and on the other hand, the level of performance by the user, the provider and the components of the cloud affect the security. They both affect the customer's confidence in the adoption of the cloud, and this is an indicator that affects the development and progression of the cloud and the light of this, the level of security and performance is considered a challenge and an obstacle to the progress and development of cloud computing.

\section{FURTHER RESEARCH}

The cloud computing is progressing but there are several factors and challenges to the user adoption of the cloud computing technology. Therefore, Technology Acceptance Model (TAM) will be taken as a model in the creation of the research model to show the user's adoption of cloud computing as in Figure (5). Performance and security are regarded as basic factors and challenges that affect the user's adoption of cloud computing technology.

H1: Security affects performance which depends on a high or low security level.

The impact of the level of security by the user and the provider; in case of high-level security, this leads to raise the level of performance of the cloud computing, where the security level depends on external factors, including the security risks, the confidentiality of data, service level agreement and physical security.

$\mathrm{H} 2$ : The level of performance by the service provider affects the level of security.

in case of high-level performance, this leads to the lifting the security level of the cloud computing, where that performance level depends on external factors such as security threats, data confidentiality, laws and regulations, service level agreement, and communication line . 
Security is regarded a major challenge in adopting cloud computing; it is the main barrier that prevents companies from the adoption of cloud computing. In fact, a cloud with unsafe infrastructure can lead to problems between the adopter and specialists such as customers, partners, providers or even the government [32]. The level of service in the cloud computing is important because the service level scale is based on the performance level. If the level of performance is high, it is a sign to the speed and quality of service, leading to user satisfaction and adoption of the cloud to make use of its electronic service [33].

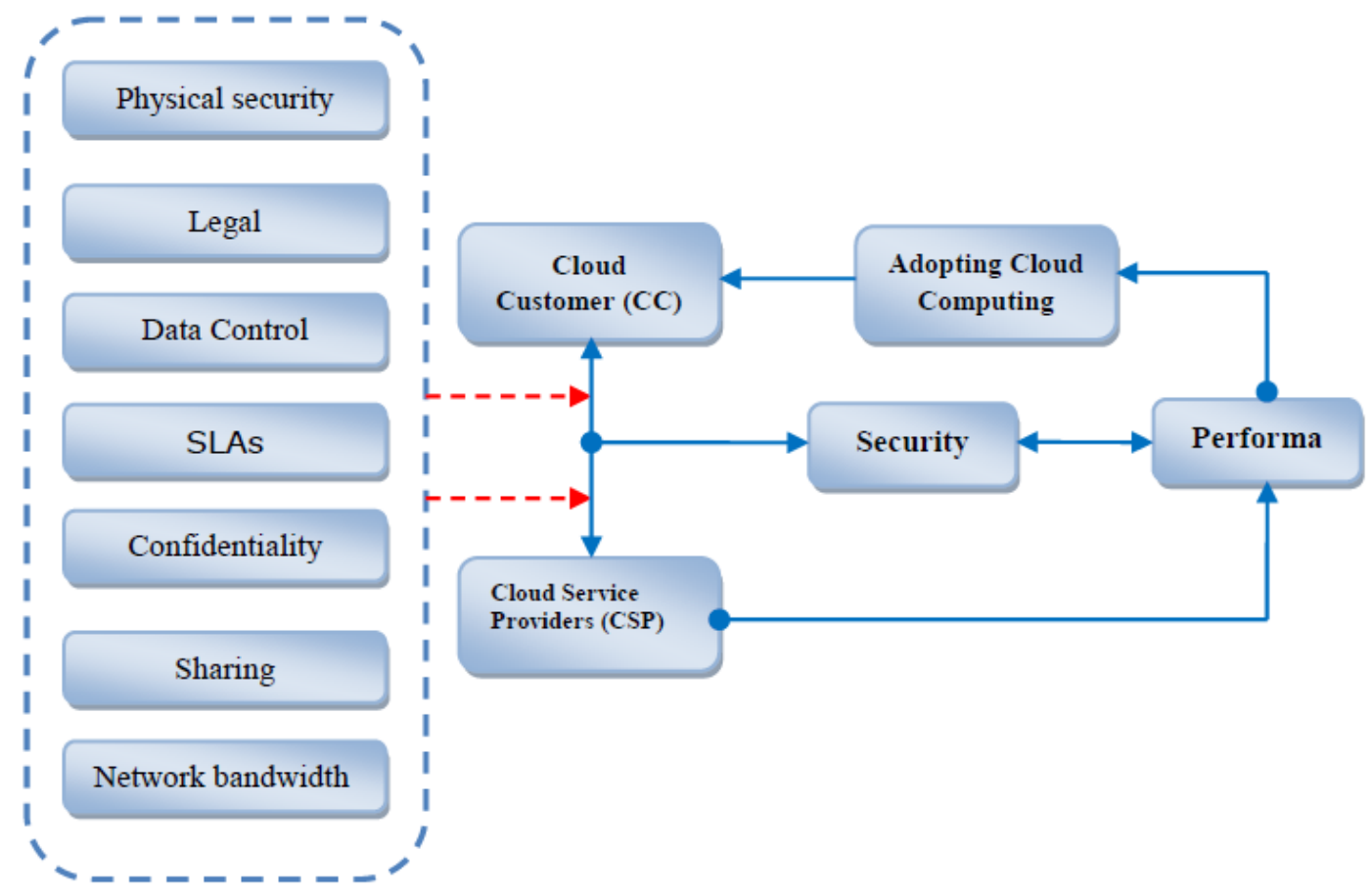

Figure 5. Research model

\section{REFERENCES}

[1] Dev, R Pande. \& Gauri Sh Kushwaha, (2015) "Cloud Computing for Digital Libraries in Universities", (IJCSIT) International Journal of Computer Science and Information Technologies, Vol. 6, No. 4, pp 3885-3889.

[2] Swati Gupta \& Kuntal Saroha, Samiksha Mehta, (2011) "Distributed Computing: An Emerging Paradigm for New Technologies", IJCSMS International Journal of Computer Science and Management Studies, Vol. 11, pp 155-164.

[3] Judith Hurwitz \& Alan Nugent, Fern Halper, (2013) Marcia Kaufman, Big Data For Dummies, Social Network, John Wiley \& Sons.

[4] Four Key Elements of a Secure, High-Performance Hybrid Cloud Networking Strategy Delivering an optimized bridge to the cloud with Citrix Cloud Bridge, Cloud Bridge White Paper 2012. https://www.citrix.com.

[5] Secure, private, and trustworthy: enterprise cloud computing with Force.com, White Paper 2013.

[6] Malvinder Singh Bali \& Shivani Khurana, (2013)" Performance Analysis of Cloud Computing under the Impact of Botnet Attack", Proceedings in International Conference on Recent Trends in Communications and Computer Network.

[7] Anurag S. Barde, (2013) “Cloud Computing and Its Vision 2015!!” International Journal of Computer and Communication Engineering, Vol. 2, No. 4.

[8] Chang, V, "Delivery and Adoption of Cloud Computing Services in Contemporary Organizations "Information and communication sciences", IGI Global, 2015.

[9] Gupta \& Manish, "Handbook of Research on Emerging Developments in Data Privacy “, IGI Global, 2014. 
[10] Bautista L., Abran A. and April A., (2012) "Design of a Performance Measurement Framework for Cloud Computing", Journal of Software Engineering and Applications, Vol. 5, No. 2, pp 69-75.

[11] Giuseppe Aceto \& Alessio Botta, (2013)" Cloud monitoring: A survey" Elsevier computer network, Vol. 57, No.9, pp 2093-2115.

[12] Niloofar Khanghahi \& Reza Ravanmehr, (2013)" Cloud Computing Performance Evaluation: Issues And Challenges", International Journal on Cloud Computing: Services and Architecture (IJCCSA), Vol.3, No.5.

[13] Shailesh Paliwal, (2014) "Performance Challenges in Cloud Computing", https://www.cmg.org

[14] Amid, K Bardsiri \& Seyyed, M Hashem, (2014) "QoS Metrics for Cloud Computing Services Evaluation", International Journal of Intelligent Systems and Applications, Vol. 6, No. 12, pp 27-33.

[15] Elisabeth Stahl \& Lydia Duijvestijn and others, (2012) "Performance Implications of Cloud Computing", ibm.com/Redbooks pp 4875.

[16] Danilo Ardagna \& Giuliano Casale and others (2012), "Quality-of-service in cloud computing: modeling techniques and their applications", Journal of Internet Services and Applications a Springer, Vol.5, No. 11.

[17] Technical Report ,“CLOUD; SLAs for Cloud services”,ETSI TR 103 125 V1.1.1 (2012), http://www.etsi.org/

[18] Cloud computing in higher education a guide to evaluation and adopting- Cisco 2010. https://www.cisco.com/web/strategy/docs/education/Cloud_Computing_in_Higher_Education.pdf

[19] Shirley Radack, (2012) "Cloud Computing: A Review of Features, Benefits, and Risks, and Recommendations for Secure, Efficient Implementations". http://www.itbusinessedge.com

[20] April Reeve, (2013) "Managing Data in Motion: Data Integration Best Practice Techniques", Elsevier Science Technology.

[21] Avinash R. Dhok \& Ashwini P. Kolhe, (2015) "A Survey on Scalable Data Security and Load Balancing in Multi Cloud Environment", IJIRST -International Journal for Innovative Research in Science \& Technology, Vol. 1 , No. 8, pp 232:236.

[22] Sherin Sreedharan \& G.Kalpana, (2013) "Security Issues and Solutions for Cloud Computing", International Journal of Computer Trends and Technology (IJCTT), Vol.4, No.4, pp 494-498.

[23] Nir Kshetri, (2013) "Privacy and security issues in cloud computing: The role of institutions and institutional evolution", Telecommunications Policy, vol. 37, No. 4, pp 372-38.

[24] Stefan Frey \& Claudia Luthje, and Christoph Reich, (2013) "Key Performance Indicators for Cloud Computing SLAs", the Fifth International Conference on Emerging Network Intelligence, pp 60-64.

[25] Safiya Okai \& Mueen Uddin and Amad Arshad, Raed Alsaqour, Asadullah Shah, (2014) "Cloud Computing Adoption Model for Universities to Increase ICT Proficiency”, SAGE Open, Vol.4, No.3, pp 1-10.

[26] K. Vaitheki, (2014) "A SLA violation reduction technique in Cloud by Resource Rescheduling Algorithm (RRA)", International Journal of Computer Application and Engineering Technology Vol. 3, No.3, pp. 217-224.

[27] Danilo Ardagna \& Giuliano Casale and Michele Ciavotta, Juan F Pérez and Weikun Wang, (2014) "Quality-of-service in cloud computing: modeling techniques and their applications", Journal of Internet Services and Applications springeropen.com, Vol. 5, No.11, pp 1-17.

[28] Niloofar Khanghahi \& Reza Ravanmehr, (2013)" cloud computing performance evaluation: issues and challenges", International Journal on Cloud Computing: Services and Architecture (IJCCSA), Vol.3, No.5,pp 29-41.

[29] Sara Qaisar \& Kausar Fiaz Khawaja, (2012) "Cloud Computing: Network/Security Threats and counter measures", Interdisciplinary Journal of Contemporary Research in Business, ijcrb.webs.com, January, Vol. 3, No.9, pp 1323 - 1329.

[30] Zaigham Mahmood, (2014) "Cloud Computing: Challenges, Limitations and R\&D Solutions", Springer.

[31] Heru Susanto \& Mohammad Nabil Almunawar and Chen Chain Kang, (2012) "Toward Cloud Computing Evolution: Efficiency vs Trendy vs Security", Computer Science Journal.

[32] M. Z. Nkhoma and D. P. T. Dang, (2013) "Contributing Factors of Cloud Computing Adoption: a Technology-Organization-Environment Framework Approach," International Journal of Information Systems and Engineering (IJISE), Vol. No.1, pp. 38- 49.

[33] Tobias Ackermann, (2012)" IT Security Risk Management: Perceived IT Security Risks in the Context of Cloud Computing”, Springer Science \& Business Media. 


\section{AUTHOR}

Dr. Nabeel Mohammed Zanoon, He received his $\mathrm{PhD}$ in Computer Systems Engineering, from South West State University, Kursk, Russia, in 2011. He is faculty member with Al-Balqa' Applied University since 2011; where he is currently Assistant professor and Head of the Department of Applied Sciences as well as Director of the ICDL Computer Centre and Cisco Academy Branch of Aqaba University College. He has published several research in several areas, Security of E-Banking, Algorithm Scheduling in Grid and Cloud, Meta-Grammar, Hardware and Architecture , Fiber

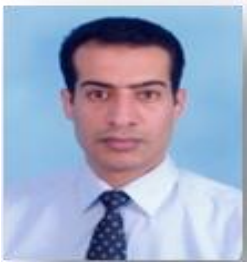
optical , Mobile Ad Hoc Networks. 